\title{
Opinião de escolares e educadores sobre saúde: o ponto de vista da escola pública de uma região periférica do Município de São Paulo
}

\author{
Opinions of students and educators concerning \\ health: the public school perspective \\ in a peripheral area of the city of São Paulo
}

Cássia B. Soares 1

Marina de G. Salvetti 2

Lívia K. de Ávila 1

\footnotetext{
1 Departamento Enfermagem em Saúde Coletiva, Escola de Enfermagem, Universidade de São Paulo. Av. Dr. Enéas de Carvalho Aguiar 419, São Paulo, SP 05403-000, Brasil. cassiaso@usp.br 2 Hospital do Câncer. Rua Prof. Antonio Prudente 211, São Paulo, SP 01509-900, Brasil.
}

\begin{abstract}
This study focused on prevailing concepts in society concerning adolescents' health problems and needs. Based on concerns in the field of Collective Health in relation to public policies for social protection, the study attempted to grasp the public school perspective concerning these issues. The study's theoretical reference was the social determination of the health-disease process and the view that health needs are translated into demands for changes in the harmful processes resulting from relations in the production process. Data were gathered through interviews with pedagogical coordinators and focus groups with adolescents in public schools from the Raposo Tavares School District (Municipality of São Paulo). The results showed that the participants recognized: (1) the sphere of social determinants at the base of adolescents' health problems and (2) in addition to disease processes, a set of social problems impacting the health-disease process, highlighting drug use and violence. Health needs are translated as demands for cross-sector public social policies.
\end{abstract}

Key words Adolescent; Teen Health; Schools; Public Policy

Resumo Este estudo tomou como objeto as concepções vigentes na sociedade sobre os problemas e necessidades de saúde dos adolescentes. Com base nas preocupações da saúde coletiva com as políticas públicas de proteção social, objetivou apreender a opinião da escola pública sobre esses temas. Referenciou-se na teoria da determinação social do processo saúde-doença e na visão de que as necessidades de saúde se traduzem em demandas de transformação dos processos nocivos conseqüentes às relações de produção. A apreensão dos dados foi realizada mediante entrevistas com Coordenadores Pedagógicos e grupos focais com adolescentes das escolas públicas do Distrito de Raposo Tavares (Município de São Paulo). Os resultados mostraram que os participantes reconheceram: (1) a esfera dos determinantes sociais na base dos problemas de saúde dos adolescentes; (2) além dos processos mórbidos, um conjunto de problemas sociais que repercutem sobre o processo saúde-doença, destacando o uso de drogas e a violência. As necessidades de saúde se traduziram em demandas por políticas sociais públicas intersetoriais.

Palavras-chave Adolescente; Saúde do Adolescente; Escolas; Políticas Sociais 


\section{Introdução}

Este trabalho toma como objeto as concepções vigentes na sociedade sobre os problemas e necessidades de saúde dos adolescentes. Integra as preocupações presentes na abordagem do tema sob a perspectiva do campo da Saúde Coletiva, que tem se valido da interdisciplinaridade para estudar o seu objeto e propor políticas socais públicas baseadas na intersetorialidade.

Em nosso país a preocupação social e pública com a infância e a adolescência veio crescendo desde a década de 80 , quando se deflagrou o processo de constituição das políticas públicas e que especificamente confluiu para a promulgação do Estatuto da Criança e do Adolescente (ECA), em 1990 (Brasil, 1991). O ECA se instituiu tendo por princípio uma inovação estabelecer a criança e o adolescente como sujeitos de direito.

A política social pública surgiu assim como resultado de forças sociais atuantes, firmado pelo compromisso público do Estado com um projeto de desenvolvimento de um determinado modelo de organização e funcionamento da sociedade (Bianchetti, 1997). A Constituição de 88 apontou, portanto, na direção de um modelo de promoção de políticas públicas que permitisse à população vulnerável socialmente pela posição ocupada nas relações entre capital e trabalho - a garantia de direitos consensuados naquela correlação de forças, fugindo da lógica assistencialista e universalizando direitos sociais. No entanto, a partir de 90, com a adesão incondicional do Estado brasileiro ao projeto neoliberal, o caráter inclusivo das políticas sociais públicas preconizadas pela Constituição - traduzido nos princípios do bem-estar social e, no caso particular da saúde, no ideário da reforma sanitária - veio sendo retirado de cena.

Contudo, setores da sociedade civil organizada têm-se mobilizado intensamente em torno da integração da juventude como objeto das políticas sociais públicas, no sentido do aperfeiçoamento não somente das práticas, mas da compreensão a respeito dos problemas enfrentados na adolescência, seja sob a ótica dos serviços, seja sob a ótica dos sujeitos sociais. Nessa interface, destaca-se aqui um conjunto de produções recentes que chamam a atenção para a teia complexa que enreda os problemas e necessidades do jovem (Abramo, 1994; Abramo et al., 2000; Ayres \& França Jr., 1996; Carlini-Cotrim et al., 2000; CNDP, 1998; Fausto \& Cervini, 1992; Galduróz et al., 1997; Guerra, 1998; Minayo \& Assis, 1994; Sposito, 1994; Zaluar, 1996).
Valendo-se do pressuposto que as adolescências vividas em contextos de carência social poderiam vulnerabilizar para específicos problemas de saúde, ou que afetam a saúde, e determinar diferentes necessidades de saúde (Soares et al., 2000, 2001), observamos que, num espaço periférico da Cidade de São Paulo, adolescentes inscritos em escolas públicas, cujas famílias apresentavam padrões desiguais de reprodução social, expressavam em freqüências diversas seus problemas e necessidades de saúde. Foi possível associar taxas maiores de ferimentos, problemas agudos respiratórios, gastrointestinais, gênito-urinários e de pele à exclusão social. A maior taxa de ocorrência de problemas crônicos de saúde, representados pelas afecções do trato respiratório, estava também entre os excluídos sociais. Foi também entre os excluídos sociais que apareceu a maior incidência de consumo excessivo de álcool. Foi a necessidade de acolhimento/proteção na família, na escola e no bairro que melhor traduziu as vulnerabilidades dos adolescentes, independentemente de sua inserção social. Porém, a necessidade de obter orientações sobre drogas, AIDS e sexualidade era tanto mais expressiva quanto mais se mergulhava na exclusão social.

No pressuposto de que a apreensão de dados trabalhados com uma abordagem qualitativa deveria complementar a análise e com o intuito de contribuir para o avanço da produção de conhecimentos e práticas comprometidos com a defesa dos direitos dos adolescentes, este trabalho objetivou reconhecer as concepções vigentes sobre os problemas e necessidades de saúde dos adolescentes.

A escola pública foi tomada como referência, reconhecendo a importância de análises que apontam para a precariedade dessa instituição social como agência de socialização dos adolescentes na sociedade contemporânea. Tal "fraqueza" vem sendo associada, em primeiro lugar, ao descaso do Estado contemporâneo afeto ao projeto neoliberal - com a educação daqueles que não têm poder de compra, produzindo as conseqüências inerentes à escola da "cesta básica" (Gentili, 1996). Em segundo lugar, a "fraqueza" da escola tem sido associada às tendências impostas pelo "modo de vida" atual, em que se acirra a desconsideração dos jovens para com as experiências dos "mais velhos", contestadas na incongruência entre o que é apregoado e o que é, de fato, realizado (Forrester, 1997; Morin, 1997).

Os jovens encontram-se ao sabor do mercado globalizado, que hoje se consolida como grande regulador das relações entre o capital e 
o trabalho e, portanto, das escolhas profissionais. A formação escolar, completamente submetida a essa lógica, vê como horizonte o trabalho terceirizado, flexível e autônomo (Frigotto, 1995). Os jovens assistem à "queda das profissões", costumeiramente apresentadas como valorosas, questionando a necessidade da educação formal como perspectiva de inserção social (Bock, 2000; Martins, 2000).

\section{Metodologia}

\section{Local de estudo}

O Distrito Administrativo (DA) Raposo Tavares, local de realização da pesquisa, fica na periferia Oeste do Município de São Paulo. A população, em 2000, era de 90.580 habitantes, havendo crescimento demográfico nos últimos dez anos. Uma pesquisa que classificou os 96 DA de São Paulo, segundo a exclusão social, revelou que Raposo Tavares ocupava a 32a posição no ranking de exclusão social. Essa classificação se deve, entre outros motivos, à situação dos 20.171 chefes de famílias, uma vez que 1.119 $(5,5 \%)$ estavam sem renda e apenas $2,36 \%$ apresentavam rendimentos acima de 20 salários mínimos (Sposati, 1996). De acordo com dados do Programa de Aprimoramento das Informações de Mortalidade no Município de São Paulo/Fundação Sistema Estadual de Análise de Dados (PROAIM/SEADE), as principais causas de mortalidade nesse distrito, em 1999, foram os problemas circulatórios (87 casos), as neoplasias (56 casos) e as causas externas, com 45 casos, sendo que $66,66 \%$ das mortes por causas externas foram por causa de homicídios. Particularmente, as mortes dos jovens dessa região foram, em 1997, majoritariamente atribuídas às chamadas causas externas. Em 1996, 1.787 adolescentes se encontravam fora da escola, o que representava $15,7 \%$ do total de adolescentes da região (Soares et al., 1998).

\section{Fontes de informação}

Tomaram-se como referência os depoimentos obtidos em 12 escolas públicas de Ensino Fundamental e Ensino Médio (antigos 1o e 2o Graus) do DA Raposo Tavares e do seu centro de juventude, resultado de entrevistas com coordenadores pedagógicos e grupos focais com adolescentes escolares (de 12 a 18 anos incompletos).

\section{Trabalho de campo}

Foram realizadas 14 entrevistas com 12 coordenadores pedagógicos das instituições escolares e dois coordenadores do Centro de Proteção ao Adolescente e do Centro de Juventude. Para que se criasse um clima de respeito e sensibilização, foram utilizados como referência os fundamentos da vertente emancipatória da pesquisa (Saul, 1985), usando um roteiro que procurava levantar questionamentos e motivações aos respondentes para fazer uma avaliação crítica da realidade pesquisada (Haguette, 1992). Assim, os objetivos da pesquisa foram explicados, tratando de pontuar aos participantes coordenadores que as preocupações do campo da saúde coletiva não se restringem aos problemas de saúde propriamente ditos, mas ampliam-se para o entendimento de suas raízes. O ECA foi mencionado no percurso das entrevistas como um marco legal que preconiza políticas de proteção à saúde do adolescente. As questões do roteiro de entrevista se referiam: aos principais problemas de saúde ou que afetam a saúde dos adolescentes que freqüentam a escola; às causas desses problemas; às ações da instituição diante desses problemas; à relação da instituição com outras do bairro; ao que consideram necessário para que os jovens tenham uma vida saudável; ao papel das instituições para satisfazer essas necessidades e efetivar os direitos preconizados pelo ECA.

Constituíram-se 21 grupos com cerca de 8 adolescentes cada um, mantendo-se uma proporcionalidade entre o número de grupos e o total de adolescentes inscritos na instituição. Os grupos foram compostos mediante escolha aleatória, pelo sorteio computacional dos números constantes nas listas de alunos matriculados nas várias séries das escolas. O contato com os adolescentes realizou-se em sala de aula, ocasião em que se esclareceu a finalidade da pesquisa. A fim de propiciar aos adolescentes um tempo para pensar sobre o tema e a oportunidade no grupo de fazer conexões entre seus problemas de saúde e as condições sociais do distrito, com base nas considerações de especialistas em grupos focais que se valem de uma abordagem emancipatória (Padilla, 1993; Zeller 1993), a escolha do distrito como locus de pesquisa foi então justificada pelos resultados da pesquisa de Sposati (1996) que localiza o distrito como um espaço que manifesta diferentes e múltiplas situações de exclusão social. Em seguida, foi-lhes apresentado um roteiro que indagava sobre: sua situação de saúde atual; os principais problemas relacionados à saúde dos adolescentes do bairro; as causas 
desses problemas; e o que consideravam necessário para ter uma vida saudável.

\section{Sistematização e análise das informações}

A sistematização dos depoimentos, gravados e transcritos, foi realizada segundo a orientação de Bardin (1977), buscando, num primeiro momento, marcar os núcleos de registro (temas) que se evidenciaram nas transcrições e, num segundo momento, avaliar aqueles que mais freqüentemente foram abordados nos grupos e entrevistas. Tomando então por base a teoria que referencia este trabalho, em seguida, os temas relativos aos problemas de saúde foram reunidos e analisados de acordo com a concepção da determinação social do processo saúdedoença, conforme mantivessem correspondência com a esfera dos determinantes ou dos resultados (Breilh, 1991, 1995). Os temas relativos às necessidades de saúde foram analisados conforme a concepção de que as necessidades de saúde "não se deduzem de uma lista fixa de consumos mínimos toleráveis, mas das demandas que aparecem para transformar os processos nocivos que surjam como conseqüências das relações de produção" (Breilh, 1991:176). Em particular, essas demandas foram analisadas conforme mantivessem correspondência com a esfera da produção de serviços de saúde ou intersetorial. A coleta de dados junto às escolas foi realizada entre os meses de setembro a dezembro de 1998 e, junto ao Centro de Juventude, nos meses de fevereiro e março de 1999, após autorização das instâncias oficiais responsáveis pelas instituições estudadas. Contactadas as instituições para agendar os grupos focais com os adolescentes, estas se responsabilizaram pelos procedimentos administrativos concernentes à participação na pesquisa.

\section{Resultados}

\section{Os problemas de saúde:}

a esfera dos determinantes

Quando solicitados a falar sobre os problemas relacionados à saúde, os adolescentes mencionaram com prontidão e majoritariamente temas como desemprego, condições precárias do bairro, entre outros, intrinsecamente vinculados aos determinantes do processo saúdedoença. Mas a precariedade dos serviços de saúde e a dificuldade de acesso foi também lembrada.

Sugeriu-se também a existência de relações entre a realidade da vida escolar e a saúde-do- ença. Os adolescentes trataram, assim, das deficiências da educação pública, insuficiente para integrá-los no ensino de 3o grau, da merenda, que consideravam inadequada, da indisponibilidade da direção das escolas para ouvir os alunos e dos recursos, por vezes existentes, mas subtilizados.

Entre os coordenadores pedagógicos, as preocupações em torno das raízes do processo saúde-doença focalizaram-se especialmente na situação da escola, embora o desemprego dos chefes de famílias tenha sido mencionado. A falta de perspectivas dos estudantes aparece como um problema social.

É preciso destacar que o tema da família foi expresso em grande freqüência pelos coordenadores pedagógicos, filiados a uma concepção de que se trata de uma agência de socialização em processo de "desestruturação irreversível”. Tal concepção, explicitamente colocada como um problema, ficou implícita quando a discussão tratava das drogas e da gravidez na adolescência, a família sendo tratada de forma negativa e culpabilizada pelos problemas dos adolescentes.

\section{Os problemas de saúde: a esfera dos resultados}

\section{- As doenças corriqueiras}

Os jovens e os coordenadores pedagógicos abordaram ainda, sem muita ênfase, aspectos mórbidos que apareciam sob a denominação de gripes, problemas de pele, dentários, doenças venéreas, AIDS, câncer.

\section{- Uso de drogas}

Os temas que mereceram destaque no âmbito dos resultados do processo saúde-doença foram o uso de drogas, a violência, os problemas comportamentais e a gravidez na adolescência. $\mathrm{Pa}-$ rece assim que os resultados foram lidos como expressões de desgaste que não necessariamente se localizam "no corpo biológico".

No que se referiu ao uso de drogas, o cigarro foi preponderantemente mencionado pelos adolescentes, apontando possíveis diferenças entre cigarro e maconha, talvez até porque o uso da maconha adquirisse o padrão de um hábito considerado "normal" entre eles. Mostraram-se especialmente preocupados com o uso do cigarro e, como no caso de outras drogas - deixando transparecer diferentes associações motivacionais e suas diversas conseqüências -, procuravam explicar a razão pela qual fumavam e os males trazidos pelo tabagismo. 
Assim como para os adolescentes, o uso de drogas foi freqüentemente problematizado pelos coordenadores, associando-o à falta de lazer e às condições de vida dos jovens. Note-se que o alcoolismo foi considerado de maneira específica, relacionado mais freqüentemente aos pais.

\section{- A violência}

O problema da violência foi relacionado à família, à escola e ao bairro, este lembrado pelos participantes como um ambiente perigoso e dominado pelo tráfico de drogas, pela convivência com brigas, tiroteios e homicídios. Mas também lembraram que a violência entre os próprios adolescentes era um fato corriqueiro em suas vidas.

\section{- Os problemas comportamentais e emocionais}

Nas manifestações dos coordenadores pedagógicos foram reconhecidas preocupações com o desgaste emocional dos jovens, expresso em comportamentos considerados problemáticos.

\section{- A gravidez na adolescência}

A discussão sobre a gravidez na adolescência só apareceu depois que os adolescentes foram estimulados pelo coordenador do grupo (constituiria ou não um problema para eles?). A gravidez não parecia estar classificada como um problema de saúde; implicava um processo de escolha. Tratavam do tema como algo comum, "natural", às vezes como irresponsabilidade; pronunciavam-se a respeito das dificuldades enfrentadas pelas garotas, que, nas suas opiniões, assumiam a maior parte das conseqüências, quase sempre sem apoio do parceiro e dos pais.

A gravidez na adolescência apareceu na fala dos coordenadores relacionada à falta de informação sobre contracepção e à falta de comunicação e atenção no âmbito familiar. Eles se preocupam também com o abandono da escola.

\section{Necessidades de saúde}

\section{- A esfera da produção de serviços de saúde}

Para se ter uma vida saudável, os participantes opinaram na direção de que seriam necessárias intervenções não somente sobre os resultados do processo saúde-doença, mas também sobre os determinantes. No âmbito mais específico dos resultados trataram então da necessidade de serviços de saúde.

Coordenadores e adolescentes identificaram também a necessidade de programas de prevenção estruturados em torno de informações sobre as doenças e outros problemas que afetam a saúde.

\section{- A esfera da intersetorialidade}

Os coordenadores pedagógicos estruturaram seus depoimentos mencionando a necessidade de um trabalho conjunto entre as agências de socialização e outras instituições sociais do bairro. Note-se que, em seus depoimentos, apesar de preconizar ações intersetoriais, testemunhavam a inexistência dessas práticas; no melhor dos casos, davam conta de que os moradores do bairro podiam ocupar a quadra da escola.

Dentre as atividades que a escola deveria realizar para ajudar a suprir as necessidades de saúde dos adolescentes, as atividades extracurriculares de caráter cultural e esportivo se destacaram entre os coordenadores.

Ainda na esfera da inter-relação entre diferentes setores da sociedade, os adolescentes lembraram da necessidade de um "bom relacionamento familiar", citando também o cuidado com o meio ambiente, evitar as drogas, melhorar as condições de vida, boas amizades, segurança e não esquecendo ainda da necessidade de trabalho entre os familiares.

\section{Discussão}

O monitoramento dos perfis epidemiológicos, proposto na perspectiva da superação dos padrões saúde-doença dos diferentes grupos sociais, tem como base o acompanhamento de indicadores relativos à reprodução social (determinantes) e não somente de indicadores que retratem os perfis saúde-doença (resultados) (Breilh, 1991, 1995). Ao avaliar os temas levantados, no que se refere aos problemas de saúde dos adolescentes, verifica-se que tanto os adolescentes quanto os coordenadores pedagógicos estavam atentos aos determinantes, não só daqueles elementos diretamente vinculados ao momento do consumo (áreas de lazer, condições de moradia, precariedade da escola, por exemplo), mas também daqueles relacionados à produção (desemprego dos chefes de família por exemplo).

O peso do infortúnio dos jovens foi atribuído sobretudo à perda da estrutura familiar tradicional, mas também à negligência do Estado, 
talvez por ser a família uma instituição visível, próxima, com possibilidade de ser fotografada na sua expressão cotidiana. No entanto, os determinantes macroestruturais, que alteram as formas de reprodução social das famílias e sua estrutura, a globalização econômica e o ajuste estrutural - componentes essenciais do modelo neoliberal, que estão favorecendo o processo de empobrecimento e exclusão social -, foram vistos de maneira longínqua e parcelar, sem que isso conduzisse a um eixo causal claro.

Os resultados do processo saúde-doença enfatizados diziam menos respeito às expressões de desgaste no corpo biológico (Salum et al., 2001) propriamente dito (problemas respiratórios, dentários e de pele, entre outros) do que a um conjunto de problemas sociais que têm repercussões sobre o processo saúdedoença dos adolescentes de maneira mais ampla (drogas, violência, problemas comportamentais, gravidez na adolescência).

Tanto coordenadores pedagógicos quanto adolescentes ponderaram sobre a existência de uma correlação entre drogas e violência, caracterizando o bairro como um espaço violento, que restringe a circulação das pessoas e a utilização das poucas áreas de lazer existentes. Essa relação tem sido associada ao caráter ilícito de algumas drogas, que acaba por vulnerabilizar os jovens de bairros pobres pela exposição perversa ao tráfico (Zaluar, 1996).

De outro lado, mesmo que corriqueiramente o uso de álcool chame menos a atenção da sociedade do que o uso de drogas ilícitas, os resultados de estudos em São Paulo mostram que os jovens estão bastante envolvidos com o uso prejudicial de álcool. Dos 6.940 jovens que morreram por causas externas no ano de 1994, 3.470 estavam alcoolizados (Carlini-Cotrim, 2000). Em 1997, 74,1\% dos estudantes de escolas públicas de Ensino Fundamental e Médio haviam referido o uso de álcool alguma vez na vida, e $37,0 \%$, no último mês (Galduróz, et al., 1997).

As interpretações sobre a violência na e contra a escola também a associam à exclusão social (Severino, et al. 1992). Ainda que os especialistas na área da violência doméstica acentuem o caráter disciplinador e coercitivo dos pais ou responsáveis nas diferentes classes sociais (Guerra, 1998), a violência em casa também parece se intensificar entre os mais pobres que sofrem regularmente o desgaste inerente à preocupação constante com a sobrevivência (Santos, 1999).

A mortalidade de jovens por causas violentas vem crescendo no Brasil, e a mortalidade de jovens do sexo masculino entre 15 e 19 anos, por arma de fogo, aumentou de 14, em 1980, para 44,4 por 100.000 habitantes em 1995 (Szwarcwald \& Leal, 1998). Em São Paulo, a maior parte desses jovens é do sexo masculino entre 15 e 17 anos, vítimas de assassinatos, assiduamente sem punição, muitas vezes em situações de linchamento, variando de acordo com suas condições de vida e o bairro em que vivem (Adorno, 2000).

A ausência de políticas sociais públicas, e porque não dizer, do próprio Estado, a disseminação da idéia da "ineficácia da educação como forma de melhorar a vida” (Zaluar, 1992) e a construção de identidade por intermédio de grupos que expressam diferentes formas de sociabilidade juvenil (Sposito, 1994), articulam-se para reservar aos jovens pobres a ocupação dos espaços de rua, posições de marginalidade, mas também novas formas de organização.

A opinião sobre a gravidez na adolescência revelou preciosas distinções. Para os adolescentes significando o resultado comum de um processo de escolha, considerando-se como problemas aqueles decorrentes da gravidez - abandono da escola e da família de origem, e a opção pelo aborto. Para os educadores a gravidez na adolescência constituiria um problema sério relacionado à falta de informação sobre contracepção e à falta de comunicação e atenção no âmbito familiar.

Esse resultado é compatível com o de outros trabalhos que mostram que tanto as causas quanto as conseqüências da gravidez na adolescência são vistas e enfrentadas de diferentes formas de acordo com a inserção de classe, o que tem sido decisivo para o tratamento interdisciplinar do tema (Guimarães, 2001), havendo taxas de gravidez na adolescência mais altas entre os grupos sociais mais pobres (Camarano, 1998). Destaque-se que extensa revisão bibliográfica revela preocupação com as repercussões da gravidez semelhantes às trazidas pelos sujeitos desta pesquisa (Maia \& Ribeiro, 2001).

Ao avaliar os temas relativos às necessidades de saúde, pode-se aquilatar uma relativa preocupação da escola com a esfera da produção de serviços de saúde, levantando necessidades de serviços de saúde e de programas de prevenção. Houve também referência à falta de ações intersetoriais, expressa na necessidade de políticas sociais voltadas para a educação e para os espaços de convivência no bairro. A necessidade de atenção aos jovens excluídos do sistema escolar (Adorno, 1991), especialmente no que diz respeito à prevenção de drogas (Carlini-Cotrim et al., 2000a), tem sido apontada em outras pesquisas. 
Observou-se que a interpretação dos educadores acerca das necessidades de saúde dos adolescentes não reduziu-se à necessidade de serviços de saúde, ampliando-se na proposição de políticas públicas de proteção ao jovem em diferentes setores.

Essa compreensão sinaliza uma superação da visão que tem dominado os encaminhamentos do Estado para a satisfação das necessidades de saúde. A história das políticas sociais no Brasil tem sido marcada pela mescla de práticas assistencialistas, que não alteram estruturalmente as condições de pobreza e exclusão, práticas que privilegiam o indivíduo particular como portador de direitos, identificados pela carência de bens e serviços e práticas focalizadas, resultantes do retraimento do Estado na sua função de redistribuição da riqueza (Anderson, 1995; Bianchetti, 1997; Laurell, 1995).

\section{Considerações finais}

Conclui-se que o tratamento qualitativo dos dados complementa a análise quantitativa sobre a natureza dos problemas de saúde dos adolescentes da área, confirmando a existência de uma necessidade de proteção social a grupos de jovens pobres, moradores de espaços periféricos, pertencentes a famílias que estão submetidas às perversas leis da reprodução social no modo de produção capitalista.

A tarefa que se coloca para a escola, bem como para o setor saúde, é sem dúvida a de superar as práticas isoladas, procurando promo- ver um trabalho conjunto, intersetorial. Algumas iniciativas vêm concretizando essa integração, na perspectiva de enfrentar criticamente os problemas dos adolescentes durante seu processo de socialização (Cyrino \& Pereira, 1999; Grynszpan, 1999; Soares \& Jacobi, 2000).

No entanto, para reverter tanto a culpabilização pelos problemas de saúde quanto a responsabilização pela satisfação das necessidades dos adolescentes, atribuída aos indivíduos e suas famílias, é fundamental que seja ampliada a discussão sobre os elementos macroestruturais que estão em jogo na teia da causalidade do processo saúde-doença.

Não menor é o esforço necessário para divulgar a idéia de que as políticas sociais não devem se traduzir em práticas que constituam um fim em si. Na medida em que estão submetidas ao projeto de acumulação do capital, em suas diferentes formas, as políticas sociais públicas devem ser defendidas como meio para preservar e aperfeiçoar as condições benéficas que a elas podem estar associadas, procurando a consolidação de oportunidades construtivas de inserção social aos adolescentes.

A finalidade das práticas tanto educacionais quanto de saúde, então, não deveria estar identificada somente com a satisfação das necessidades materiais do indivíduo, mas também com as necessidades não materiais, as identificadas com a construção de um sujeito ativo, que possa desenvolver suas potencialidades, que seja capaz de participar socialmente e construir um projeto de transformação social, no caminho da emancipação humana.

\section{Agradecimentos}

Este trabalho congrega parte dos resultados do projeto matriz a que está vinculado, financiado pela Fundação de Amparo a Pesquisa do Estado de São Paulo (FAPESP) (Auxílio Pesquisa - Proc. FAPESP no 97/ 11861-2). Faz parte do Projeto Integrado de Pesquisa que recebeu auxílio financeiro da Pró-reitoria de Pesquisa da Universidade de São Paulo (USP) - projeto PII, no ano de 1998 e projeto PI, no ano de 1999. Foi apresentado no XII Congress of the International Association on Health Policy e XIV Meeting on Public Health Care Delivery Systems, Palma de Mallorca, Spain, 21 a 24 de maio de 2002. Agradecemos: à FAPESP e à Pró-reitoria de Pesquisa da USP; às Secretarias Municipal e Estadual de Educação e à Profa. Maria Josefina Leuba Salum. 


\section{Referências}

ABRAMO, H. W., 1994. Cenas Juvenis: Punks e Darks no Espetáculo Urbano. São Paulo: Scritta-Anpocs.

ABRAMO, H. W.; FREITAS, M. V. \& SPOSITO, M. P. (org.), 2000. Juventude em Debate. São Paulo: Cortez Editora.

ADORNO, S., 1991. A socialização incompleta: Os jovens delinqüentes expulsos da escola. Cadernos de Pesquisa, 79:77-80.

ADORNO, S., 2000. Adolescentes, crime e violência. In: Juventude em Debate (H. W. Abramo, M. V., Freitas \& M. P. Sposito, org.), pp. 97-109, São Paulo: Cortez Editora.

ANDERSON, P., 1995. Balanço do neoliberalismo. In: Pós-neoliberalismo: As Políticas Sociais e o Estado Democrático (E. Sader \& P. Gentili, org.), pp. 9-23, Rio de Janeiro: Paz e Terra.

AYRES, J. R. C. M. \& FRANÇA Jr., I. F., 1996. Saúde do adolescente. In: Saúde do Adulto: Programas e Ações na Unidade Básica (L. B. Schraiber, M. I. B. Nemes \& R. B. Mendes-Gonçalves, org.), pp. 6685, São Paulo: Editora Hucitec.

BARDIN, L., 1977. Análise de Conteúdo. Lisboa: Edições 70.

BIANCHETTI, R. G., 1997. Modelo Neoliberal e Políticas Educacionais. São Paulo: Cortez Editora.

BOCK, S., 2000. A inserção do jovem no mercado de trabalho. In: Juventude em Debate (H. W. Abramo, M. V. Freitas \& M. P. Sposito, org.), pp. 11-16, São Paulo: Cortez Editora.

BRASIL, 1991. Estatuto da Criança e do Adolescente: Lei 8069, de 13.07.1990. São Paulo: Editora Cortez.

BREILH, J., 1991. Epidemiologia: Economia, Política e Saúde. São Paulo: Universidade Estadual Paulista/ Editora Hucitec.

BREILH, J., 1995. Nuevos Conceptos y Técnicas de Investigación: Guía Pedagógica para un Taller de Metodología. Quito: Centro de Estudios y Asesoría en Salud.

CAMARANO, A. A., 1998. Fecundidade e anticoncepção da população jovem. In: Jovens Acontecendo na Trilha das Políticas Públicas (Comissão Nacional de População e Desenvolvimento - CNPD, org.), pp. 109-133, Brasília: CNDP.

CARLINI-COTRIM, B. H., 2000. Drogas - Estranhando o óbvio. In: Juventude em Debate (H. W. Abramo, M. V. Freitas \& M. P. Sposito, org.), pp. 71-79, São Paulo: Cortez Editora.

CARLINI-COTRIM, B. H.; GAZAL-CARVALHO, C. \& GOUVEIA, N., 2000. Comportamentos de saúde entre jovens estudantes das redes pública e privada da área metropolitana do Estado de São Paulo. Revista de Saúde Pública, 34:636-645.

CNDP (Comissão Nacional de População e Desenvolvimento), 1998. Jovens Acontecendo na Trilha das Políticas Públicas. v. 2. Brasília: CNPD.

CYRINO, E. G. \& PEREIRA, M. L. T., 1999. Reflexões sobre uma proposta de integração saúde-escola: O projeto saúde e educação de Botucatu, São Paulo. Cadernos de Saúde Pública, 15(Sup. 2):39-44.

FAUSTO, A. \& CERVINI, R., 1992. O Trabalho e a RuaCrianças e Adolescentes no Brasil Urbano dos Anos 80. São Paulo: Cortez Editora.

FORRESTER, V., 1997. O Horror Econômico. São Paulo: Editora UNESP.
FRIGOTTO, G., 1995. Educação e a Crise do Capitalismo Real. São Paulo: Cortez Editora.

GALDURÓZ, J. C. F.; NOTO, A. R. \& CARLINI, E. A., 1997. IV Levantamento sobre o Uso de Drogas entre Estudantes de 1o e 2o graus em 10 Capitais Brasileiras: 1997. São Paulo: Departamento de Psicobiologia, Universidade Federal São Paulo/ Centro Brasileiro de Informações sobre Drogas Psicotrópicas.

GRYNSZPAN, D., 1999. Educação em saúde e educação ambiental: Uma experiência integradora. Cadernos de Saúde Pública, 15(Sup. 2):133-138.

GUIMARÃES, E. M. B., 2001. Gravidez na adolescência: Uma visão multidisciplinar. Pediatria Moderna, 37(Edição Especial):29-32.

GENTILI, P., 1996. Adeus à escola pública: A desordem neoliberal, a violência do mercado e o destino da educação das maiorias. In: Pedagogia da Exclusão: Crítica ao Neoliberalismo em Educação (P. Gentili, org.), pp. 228-252, Petrópolis: Vozes.

GUERRA, V. N. A., 1998. Violência de Pais contra Filhos: A Tragédia Revisitada. São Paulo: Cortez Editora.

HAGUETTE, T. M. F., 1992. Metodologias Qualitativas na Sociologia. Petrópolis: Vozes.

LAURELL, A. C., 1995. Avançando em direção ao passado: A política social do neoliberalismo. In: Estado e Políticas Sociais no Neoliberalismo (A. C. Laurell, org.), pp. 151-178, São Paulo: Cortez Editora.

MAIA, F. F. R. \& RIBEIRO, J. G. L., 2001. Aspectos médico-sociais da gravidez na adolescência nos últimos 20 anos: Uma revisão da literatura. Revista Médica de Minas Gerais, 11:34-39.

MARTINS, H. H. S., 2000. A juventude no contexto da reestruturação produtiva. In: Juventude em debate (H. W. Abramo, M. V. Freitas \& M. P. Sposito, org.), pp. 17-22, São Paulo: Cortez Editora.

MINAYO, M. C. S. \& ASSIS, S. G., 1994. Saúde e violência na infância e na adolescência. Jornal de Pediatria, 70:263-266.

MORIN, E., 1997. Cultura de Massas no Século XX: O Espírito do Tempo - I - Neurose. Rio de Janeiro: Forense Universitária.

PADILLA, R. 1993. Using dialogical research methods in group interviews. In: Successful Focus Groups: Advancing the State of the Art (D. L. Morgan, ed.), pp. 167-183, Newbury Park: Sage.

SALUM, M. J. L.; QUEIROZ, V. M.; KOGANEZAWA, E.; FINI, A. \& FARIA, L. M., 2001. A construção de perfis epidemiológicos e a responsabilidade da universidade pública na luta pela saúde como direito social. In: Memorias del VIII Congresso Latinoamericano de Medicina Social e XI Congresso de La Asociación Internacional de Políticas de Salud, CD-ROM, La Habana: Asociación Latinoamericana de Medicina Social.

SANTOS, E. V., 1999. Para Além do Desfecho: A Violência Física Doméstica contra o Escolar como Processo Social e como Questão da Saúde Coletiva. Tese de Doutorado. São Paulo: Escola de Enfermagem, Universidade de São Paulo.

SEVERINO, A. J.; PINO, A.; SLHESSRENKO, S. M. MARTINS, J. S.; DEMASCENO, M. N.; CALAZANS, 
M. J. C.; CAMPOS, M. M. M.; KRISCHKE, P. J. D. S.; FUKUI, L.; BARRETO, V.; ZALUAR, A.; ADORNO, S.; CARVALHO, S.; PINTO, T. C. R.; GUERRA, V. N. A.; MONLEVADE, J. A. C.; MORAES, I. N.; GOHN, M. G. \& CONTI, R., 1992. Sociedade Civil e Educação, Campinas: Papirus/Centro de Estudos Educação e Sociedade/São Paulo: Associação Nacional de Educação/Associação Nacional de Pósgraduação e Pesquisa em Educação.

SAUL, A. M. 1985. Avaliação Emancipatória: Uma Proposta Democrática para Reformulação de um Curso de Pós-graduação. Tese de Doutorado. São Paulo: Psicologia da Educação, Pontifícia Universidade Católica de São Paulo.

SOARES, C. B.; ÁVILA, L. K. \& SALVETTI, M. G., 1998. Vulnerabilidade de adolescentes no SILOS - Butantã. Saúde e Sociedade, 7:63-82.

SOARES, C. B.; ÁVILA, L. K. \& SALVETTI, M. G., 2000. Necessidades (de saúde) de adolescentes do D.A. Raposo Tavares SP, referidas à família, escola e bairro. Revista Brasileira de Crescimento e Desenvolvimento Humano, 10:19-34.

SOARES, C. B.; SALVETTI, M. G. \& ÁVILA, L. K., 2001. Necessidades e problemas de saúde de adolescentes numa sociedade dual. In: Memorias del VIII Congresso Latinoamericano de Medicina Social e XI Congreso de la Asociacion Internacional de Politicas de Salud, CD-ROM, La Habana: Asociación Latinoamericana de Medicina Social.
SOARES, C. B. \& JACOBI, P. R., 2000. Adolescentes, drogas e AIDS: Avaliação de um programa de prevenção escolar. Cadernos de Pesquisa, 109:213-237.

SPOSATI, A. (org.), 1996. Mapa da Exclusão/Inclusão Social da Cidade de São Paulo. São Paulo: EDUC.

SPOSITO, M. P., 1994. A sociabilidade juvenil e a rua: novos conflitos e ação coletiva na cidade. Tempo Social: Revista de Sociologia da USP, 5:161-178.

SZWARCWALD, C. L. \& LEAL, M. C., 1998. Sobrevivência ameaçada dos jovens brasileiros: A dimensão da mortalidade por armas de fogo. In: Jovens Acontecendo na Trilha das Políticas Públicas (Comissão Nacional de População e Desenvolvimento CNPD, org.), pp. 363-393. Brasília: CNPD.

ZALUAR, A.(org.), 1992. Violência e Educação. São Paulo: Livros do Tatu/Cortez Editora.

ZALUAR, A., 1996. Da Revolta ao Crime S.A. São Paulo: Moderna.

ZELLER, R. A., 1993. Focus group research on sensitive topics: setting the agenda without setting the agenda. In: Successful Focus Groups: Advancing the State of the Art (D. L. Morgan, ed.), pp. 167183, Newbury Park: Sage.

Recebido em 9 de novembro de 2001

Versão final reapresentada em 30 de dezembro de 2002 Aprovado em 25 de abril de 2003 